\title{
Lack of correlation between sGC subunit expression and sGC activity in cerebral development is due to non-heterodimerizing subunits in vivo
}

\author{
Nadine Haase*, Monika Seeanner, Tobias Haase and Soenke Behrends
}

Address: Institut für Pharmakologie, Toxikologie und Klinische Pharmazie, TU Braunschweig, Germany

Email: Nadine Haase* - nadine.haase@tu-bs.de

* Corresponding author

from 4th International Conference of cGMP Generators, Effectors and Therapeutic Implications

Regensburg, Germany. 19-21 June 2009

Published: II August 2009

BMC Pharmacology 2009, 9(SuppI I):P20 doi:I0.II86/I47I-22I0-9-SI-P20

This abstract is available from: http://www.biomedcentral.com/I47I-22I0/9/SI/P20

(C) 2009 Haase et al; licensee BioMed Central Ltd.

\section{Background}

A number of studies have shown that the nitric oxide (NO)/cGMP signalling pathway plays a major role in neuronal cell differentiation and in the central nervous system during development, but much less is known about the expression and regulation of the different subunits of NO sensitive guanylyl cyclase (sGC) in the developing brain. In the present study, we have analysed the regulation and expression of sGC in brain of rats during postnatal development using biochemical methods.

\section{Methods}

Wistar rats at 1-4 days (neonatal) and 90-120 days (adults) old were sacrificed by $\mathrm{CO}_{2}$-asphyxia and decapitation. The cerebellum and cerebrum of neonatal and adult rats were analysed by Western blot analysis using antibodies against the sGC, by immunoprecipitation and by measurement of the specific sGC activity under basal, NO stimulated and heme independent stimulated conditions.

\section{Results}

Experiments in brain show a decrease in $\alpha_{1}$ expression and an increase in $\alpha_{2}$ expression in cerebellum of adult rats compared to neonatal rats. Enzyme activity in cerebellum shows a significant increase in NO stimulated sGC activity from adult animals compared to neonatal rats. In cerebrum, there was a similar significant increase in expression of the $\alpha_{2}$ subunit in adult animals, but there were no significant changes for the $\beta_{1}$ or $\alpha_{1}$ subunit expression. Surprisingly, NO stimulated sGC activity decreased significantly in cerebrum from adult as compared to neonatal animals. To examine sGC subunit heterodimerization, we performed immunoprecipitation experiments. These experiments show that there is partial failure of sGC subunits to heterodimerize in adult cerebrum but not in neonatal cerebrum. This explains the surprising decrease of sGC activity in adult cerebrum in spite of stable or increased expression of the sGC subunits.

\section{Discussion}

A lack of correlation between sGC protein expression and sGC activity has been first observed by Nedvetsky et al. 2002 [1] in a thorough study investigating the regional distribution of sGC in rat brain. In our study concentrating on the role of sGC in rat brain during postnatal development we found a similar striking lack of correlation between sGC expression and activity. Our data indicate that this is due to a pool of sGC subunits that fail to heterodimerize.

\section{References}

I. Nedvetsky PI, Kleinschnitz C, Schmidt $\mathrm{HH}$ : Regional distribution of protein and activity of the nitric oxide receptor, soluble guanylyl cyclase, in rat brain suggests multiple mechanisms of regulation. Brain Res 2002, 950:148-54. 